\title{
Operational results on the fully automatic LHC collimator alignment
}

\author{
Gabriella Azzopardi® ${ }^{1,2, *}$ Belen Salvachua, ${ }^{1}$ Gianluca Valentino, ${ }^{2}$ \\ Stefano Redaelli, ${ }^{1}$ and Adrian Muscat ${ }^{2}$ \\ ${ }^{1}$ CERN, Geneva 1211, Switzerland \\ ${ }^{2}$ University of Malta, Msida MSD 2080, Malta
}

(Received 13 June 2019; published 30 September 2019)

\begin{abstract}
The Large Hadron Collider has a complex collimation system installed to protect its sensitive equipment from normal and abnormal beam losses. The collimators are set around the beam following a multistage transverse setting hierarchy. The insertion position of each collimator is established using beam-based alignment techniques to determine the local beam position and rms beam size at each collimator. During previous years, collimator alignments were performed semiautomatically, with collimation experts present to oversee and control the alignment. During run II, a new fully automatic alignment tool was developed and used for collimator alignments throughout 2018. This paper discusses the improvements on the alignment software to automate it using machine learning, whilst focusing on the operational results obtained when testing the new software in the LHC. The alignment tests were conducted with both proton and ion beams, and angular alignments were performed with proton beams. This upgraded software successfully decreased the alignment time by a factor of 3 and made the results more reproducible, which is particularly important when performing angular alignments.
\end{abstract}

DOI: 10.1103/PhysRevAccelBeams.22.093001

\section{INTRODUCTION}

The Large Hadron Collider (LHC) at CERN is the largest particle accelerator in the world, used to accelerate and collide two counterrotating beams, with an unprecedented center-of-mass energy of $13 \mathrm{TeV}[1,2]$. The LHC is made up of eight arcs containing superconducting magnets and eight straight sections, referred to as insertion regions (IRs). Four IRs house the four main experiments: ATLAS (IR1) [3], ALICE (IR2) [4], CMS (IR5) [5] and LHCb (IR8) [6], which are located at the points where the beams are brought into collisions, referred to as interaction points (IPs) [7].

The LHC is susceptible to beam losses from normal and abnormal conditions, which may generate a transition from superconducting to normal conducting state (quench) of the superconducting magnets. A robust collimation system is used to safely dispose of beam losses by concentrating them in the collimation regions. This protects the superconducting magnets and other sensitive equipment from any damage that may be caused [8]. Moreover, the collimation system provides a $99.998 \%$ cleaning efficiency of all halo particles, to reduce the background for the experiments.

\footnotetext{
"gabriella.azzopardi@cern.ch
}

Published by the American Physical Society under the terms of the Creative Commons Attribution 4.0 International license. Further distribution of this work must maintain attribution to the author(s) and the published article's title, journal citation, and DOI.
An LHC collimator is composed of two parallel absorbing blocks, referred to as jaws, inside a vacuum tank. The jaws are identified as left or right, depending on their position with respect to the incoming beam. The collimators are installed with a fixed rotational angle, depending on their location and functionality, which allow to clean in either the horizontal $(\mathrm{H})$, vertical (V) or skew (S) plane. The jaws must be positioned symmetrically around the beam and their coordinate system is displayed in Fig. 1(a). Each jaw can be moved individually using two stepping motors in the jaw corners, allowing collimators to be positioned at different gaps and angles, as displayed in Fig. 1(b).

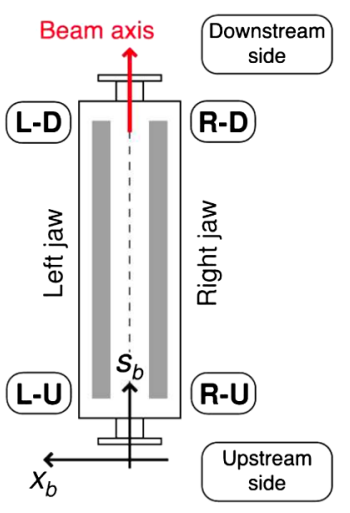

(a)

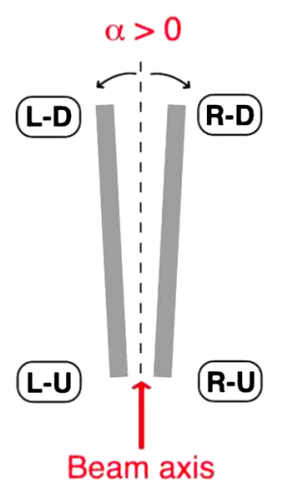

(b)
FIG. 1. (a) The collimator coordinate system and (b) the jaw tilt angular convention as viewed from above, from [9]. 
The maximum possible operational angle in either direction is $1900 \mu \mathrm{rad}$ [10]. The jaw corners are known as left-up (LU) and right-up (RU) when they are upstream of the beam and left-down (LD) and right-down (RD) when they are downstream of the beam. A fifth axis is also available to move the tank housing the jaws in the orthogonal plane, in case a region of the jaw surface would be damaged due to beam impacts. Linear variable differential transformers provide an independent measurement of these five settings, as well as the upstream and downstream jaw gap. Four resolvers count the steps of each motor, and ten switches are used to prevent the jaws from moving too far in or out.

The LHC collimation system consists of 100 collimators, which are mainly concentrated in two dedicated cleaning insertion regions; IR7 enhances betatron cleaning, and IR3, where the dispersion is larger, enhancing off-momentum cleaning [11]. Collimators provide halo cleaning using a multistage hierarchy, as shown in Fig. 2. The hierarchy is set up such that primary collimators (TCP) are placed closest to the beam to intercept the primary halo particles; the secondary collimators (TCSG) are retracted from the primary ones to clean secondary particles; the absorbers (TCLA) absorb the remaining showers; and the tertiary collimators (TCT) are further retracted from the TCSGs (but closer than TCLAs) to provide a local protection to the inner triplet superconducting magnets, which are installed around the IPs. Other collimators include the physics debris collimators (TCLs), which are further retracted from the TCTs to mitigate the impact of collision products.

In order to establish this cleaning hierarchy, the collimators must be aligned with a precision of a few tens of $\mu \mathrm{m}$, which corresponds to a beam size of $0.25 \sigma$ at $6.5 \mathrm{TeV}$ at the primary collimators. Two types of beam monitoring systems are available to align collimators.

Beam loss monitoring (BLM).-This system uses approximately 3600 ionization chambers installed around the LHC ring, to detect ionizing radiation resulting from particle losses $[13,14]$. If the detected losses exceed a predefined threshold, the BLM system is able to trigger a beam extraction to prevent damage to the machine. Each collimator has a dedicated BLM device positioned outside the beam vacuum, immediately downstream, as shown in Fig. 3(a). Such devices are used to detect beam losses generated when halo particles impact the collimator jaws.

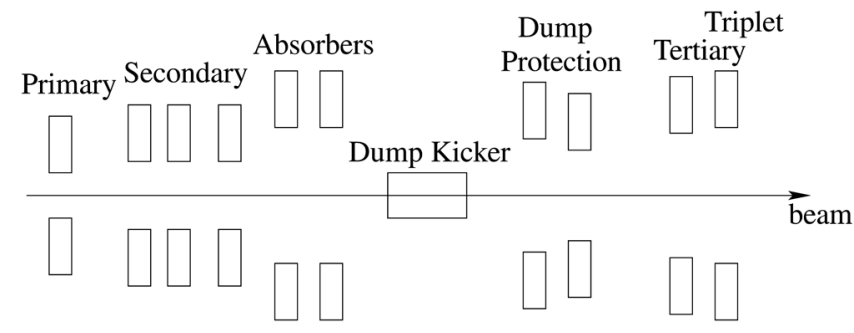

FIG. 2. Simplified sketch of the gap opening arrangement of collimator classes normalized by beam size, adapted from [12].

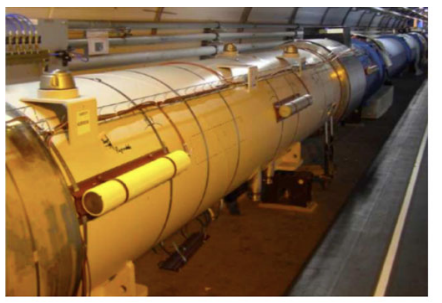

(a)

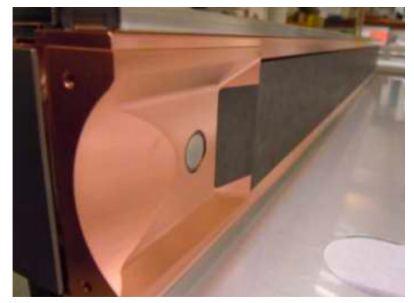

(b)
FIG. 3. (a) BLM detectors installed in the LHC and (b) BPM detectors installed in collimator jaws.

Recorded losses are proportional to the amount of beam intercepted by the collimator jaws and are measured in units of Gy/s. Dispersion suppressors (DS) aim to reduce the machine dispersion inside the insertions, therefore the losses in the DS of IR7 and IR3 are key observables to evaluate the cleaning efficiency of the system.

Beam position monitoring (BPM).-This system consists of a set of electromagnetic pickups to measure the beam position in the horizontal and vertical planes. These devices are used to control the beam trajectory within the machine, and are not located close enough to collimators to be used directly for collimator alignments. During the long shutdown of the LHC in 2013, 20 collimators have been replaced/introduced with a new design in which BPM pickup buttons are embedded in the collimator jaws [15]. These embedded BPMs are able to provide a direct measurement of the beam orbit at the collimators by analyzing the electrode signals, therefore allowing for a safer and faster alignment without requiring contact with the beam. However, BPMs are only available for less than a fifth of the collimators.

The outline of the paper is as follows. The collimation alignment specifications are introduced in Sec. II. Section III discusses the alignment evolution over the years and describes the BLM-based collimator alignment software before the full automation. Section IV gives an overview of the changes introduced for the new full automation used throughout 2018. Finally, Secs. V and VI discuss the alignment results using the new software during the proton and ion runs, respectively.

\section{ALIGNMENT SPECIFICATIONS}

Each year of operation begins with a commissioning phase which, among other activities, involves aligning all collimators and ensuring the correct hierarchy for LHC operation [16]. Collimator alignment campaigns are performed for different machine states along the LHC cycle: (i) Injection-at the $450 \mathrm{GeV}$ injection plateau, 79 collimators are aligned with BLM detectors, including the four injection protection collimators; (ii) Flattop-after both beams are ramped to $6.5 \mathrm{TeV}, 75$ collimators are aligned with BLM detectors; (iii) Squeeze-at the point of shrinking the beam size at the experimental IPs to increase the rate 
TABLE I. List of 2018 settings for collimation. The settings are expressed in beam size values, assuming a nominal beam emittance of $3.5 \mu \mathrm{m}$.

\begin{tabular}{|c|c|c|c|c|c|}
\hline Collimator & IR & Orientation & Injection $(\sigma)$ & Flattop $(\sigma)$ & Squeeze $(\sigma)$ \\
\hline Primary (TCP) & 7 & $\mathrm{H} / \mathrm{V} / \mathrm{S}$ & 5.7 & 5.0 & 5.0 \\
\hline Secondary (TCSG) & 7 & $\mathrm{H} / \mathrm{V} / \mathrm{S}$ & 6.7 & 6.5 & 6.5 \\
\hline Absorber (TCLA) & 7 & $\mathrm{H} / \mathrm{V}$ & 10.0 & 10.0 & 10.0 \\
\hline Primary (TCP) & 3 & $\mathrm{H}$ & 8.0 & 15.0 & 15.0 \\
\hline Secondary (TCSG) & 3 & $\mathrm{H} / \mathrm{V}$ & 9.3 & 18.0 & 18.0 \\
\hline Absorber (TCLA) & 3 & $\mathrm{H}$ & 12.0 & 20.0 & 20.0 \\
\hline Secondary (TCSP) & 6 & $\mathrm{H}$ & 7.5 & B1: 7.8/B2: 7.4 & 7.3 \\
\hline Dump protection (TCDQ) & 6 & $\mathrm{H}$ & 8.0 & B1: 7.8/B2: 7.4 & 7.3 \\
\hline Tertiary (TCT) & $1 / 5$ & $\mathrm{H} / \mathrm{V}$ & 13.0 & 15.0 & 9.0 \\
\hline Tertiary (TCT) & 8 & $\mathrm{H} / \mathrm{V}$ & 13.0 & 15.0 & 15.0 \\
\hline Tertiary (TCT) & 2 & $\mathrm{H} / \mathrm{V}$ & 13.0 & 37.0 & 37.0 \\
\hline
\end{tabular}

of collisions, 16 TCTs are aligned with BPM pickups as only the optics are changed; (iv) Collisions-when the beams are brought into collisions, 16 TCTs are aligned with BPM pick-ups and 12 TCLs are aligned with BLM devices, as the orbit is only changed at the experimental IPs.

Proton beams were first injected in the LHC in 2008 and are now routinely used to perform collimator alignments and to determine the beam orbit and beam size at each collimator location using the BLMs and BPMs. The beam orbit and beam size are initially unknown and may deviate from the design orbit or the collimator tank may be misaligned. This information is required to establish the hierarchy, such that the jaws are positioned within a certain number of standard deviations (beam $\sigma$ ) from the beam center, and to generate continuous setting functions for the whole LHC cycle $[17,18]$. The collimator settings used during 2018 operation at the different machine states are listed in Table I.

Over time, typically several months of operation, the beam orbit may shift due to ground motion, thermal effects and machine effects [19]. As a result, the collimation system performance is regularly monitored every three

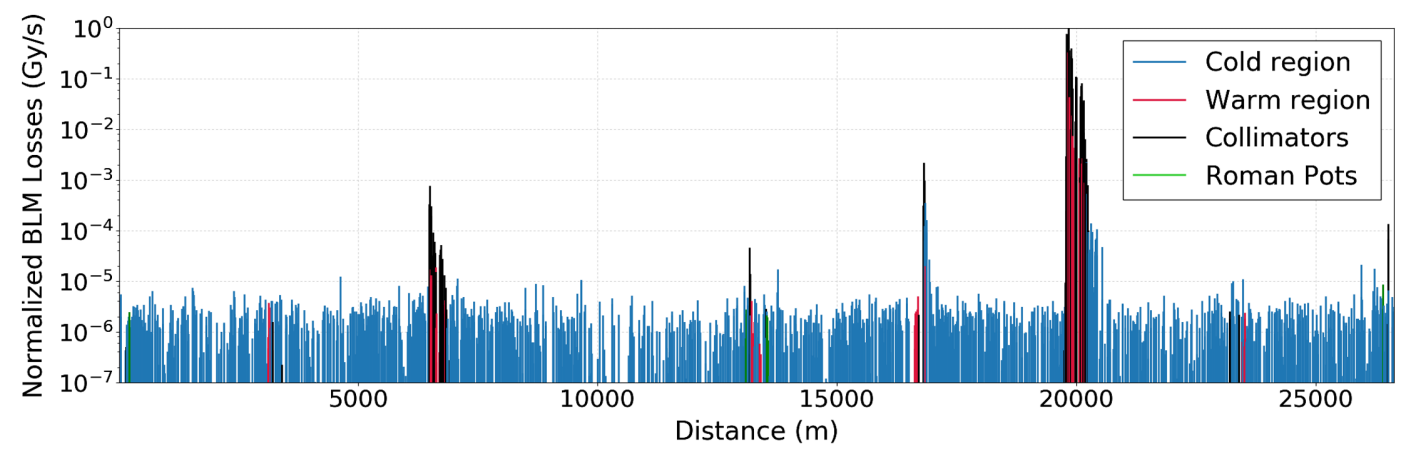

(a)

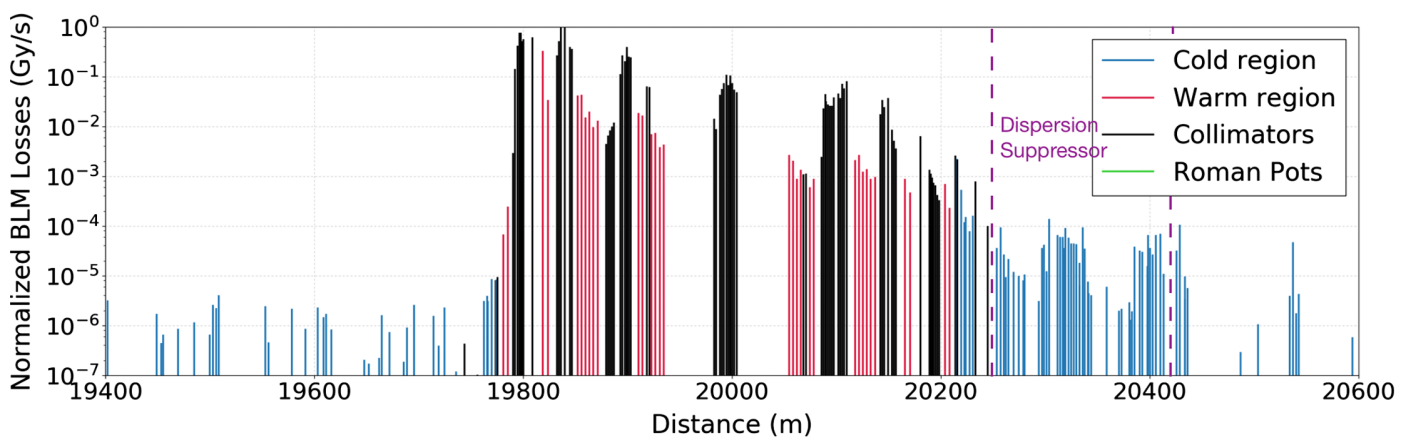

(b)

FIG. 4. An example of a beam 1 betatron horizontal loss map at $6.5 \mathrm{TeV}$, where the data points are readings taken from BLM detectors in (a) all IRs, and (b) IR7, highlighting the cleaning inefficiency in the dispersion suppressor. 
months of operation, or when the configuration is changed. This is done by inducing slow (multiturn) beam losses, such that a large number of particles are purposely sent onto primary collimators in a controlled way, and the resulting electromagnetic showers can be detected by the BLM devices around the LHC ring. A beam loss map, showing the spatial distribution of the measured losses along the LHC ring, can then be generated, as shown in Fig. 4(a), to validate the collimator setup. A zoom into IR7 is shown in Fig. 4(b), providing information on the collimation hierarchy and halo cleaning performance.

In case of a degradation in performance, the collimators might need to be realigned to generate a new setup for the current beam state. This could be observed as a degradation of the cleaning in the dispersion suppressor of IR7, or a difference in the pattern of the beam losses at the collimators. Collimator reconfiguration is also required when machine parameters are changed, such as the $\beta^{*}$, which defines the colliding beam size in the experimental points where the beams are brought into collisions, or the local orbit configurations around the IP [20].

\section{ALIGNMENT EVOLUTION}

The cumulative net time required to align the collimators over the years during all machine states (injection, flattop, squeeze and collisions) is displayed in Fig. 5, excluding any operational overhead for configurations and setups. This diagram clearly indicates the consistent decrease in the alignment time each year. The major improvements leading to collimation alignment milestones over the years include: (i) run I (2011-introduction of semiautomatic alignment software using $1 \mathrm{~Hz}$ BLM data; 2012 - availability of $12 \mathrm{~Hz}$ BLM data); (ii) run II (2015first collimators installed with BPMs; 2016-availability of $100 \mathrm{~Hz}$ BLM data; 2018-introduction of fully automatic alignment software, without parallelization of beams 1 and 2).

The initial alignment of the system in 2010 took 55 hours. Since then, several software and hardware upgrades were introduced to improve the alignment time and to reduce the complexity of the alignment procedure.

The majority of the collimators are aligned with BLM detectors, however in long shutdown 1 (before the start of run II) the first collimators were installed with BPMs. Collimators aligned with BPMs use an alignment algorithm proposed in [21], which automatically centers the collimator jaws around the beam, based on its position. The algorithm is based on successive approximation, which is required due to the nonlinear BPM sensitivity to beam displacement. This alignment procedure was already automated, therefore this section focuses on the BLM alignment procedure.

The latest BLM software upgrade is the full-automation introduced in 2018, which during commissioning aligned the collimators in the two beams sequentially, in contrast to previous years (see Fig. 5). The reason is that, in the past,

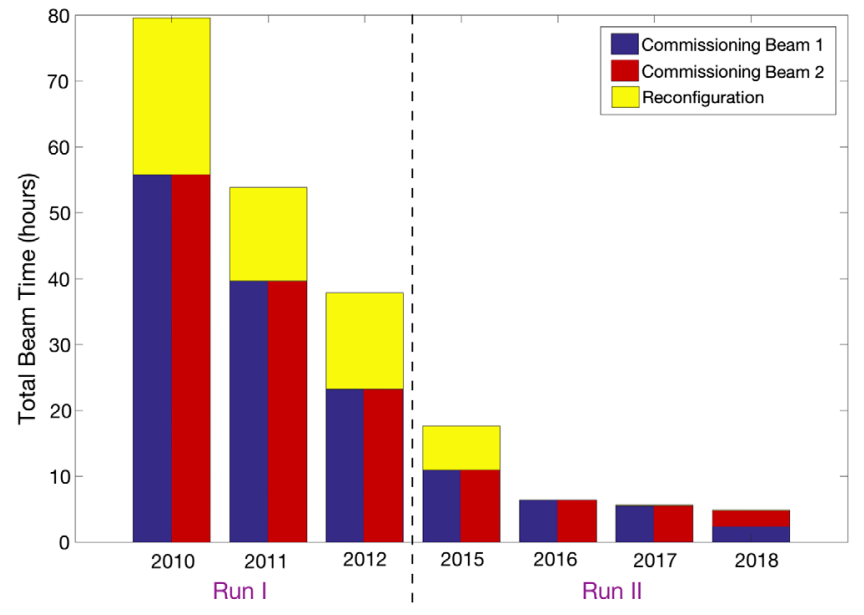

FIG. 5. The time taken to align all collimators for commissioning and any reconfigurations, during runs I and II. During 20102017 the collimators in the two beams were aligned in parallel (vertical bars), whereas in 2018 they were aligned one after the other (horizontal bars).

collimation experts would cautiously select which collimators to align. Not all collimators can be aligned in parallel, as when a collimator jaw touches the beam envelope, particle showers are propagated in the beam direction with a certain angle. This results in the possibility of cross talk, i.e., additional beam losses measured in BLM detectors at nearby collimators and also in the other beam pipe. During 2018 commissioning the full automation did not yet account for possible cross talk between the collimators therefore they were aligned sequentially, however one can still appreciate a decrease in the alignment time required.

\section{A. Overview of collimator alignment software}

Collimators aligned with BLM devices use a beam-based alignment (BBA) procedure, to determine the beam center and beam size at each collimator. This is a four-step procedure established in [22]; it was tested with a prototype collimator in the SPS $[23,24]$ and was used in the LHC from the start-up in 2010 onwards [12]. The standard sequence involves aligning a reference collimator in addition to the collimator in question (i), which is taken to be the primary collimator in the same plane as collimator $i$. This creates a "reference halo" that extends into the aperture of collimator $i$. The alignment of the collimators is beam based as a collimator's jaws are moved towards the beam while observing the spikes in the beam loss signal of its respective BLM device. A jaw is classified as aligned when a signature spike pattern is detected in the losses, indicating that it reached a transverse position closer to the beam than the reference collimator.

Prior to the full automation, the software established in $[25,26]$ was used to align collimators using a semiautomatic algorithm: (1) User selects collimator to be aligned. (2) User selects BLM threshold. (3) Start alignment: 
(a) Collimator automatically moves towards the beam. (b) Collimator automatically stops when its losses exceed the BLM threshold. (4) User analyzes the spikes in the BLM losses to check if the collimator is aligned.

This approach is time consuming and requires collimation experts to be available for the entire duration of the alignments, especially when a large number of collimators need to be aligned. The results obtained with the semiautomatic alignment software are summarized in [27], while this paper focuses on the results obtained using the fully automatic alignment software.

\section{FULLY AUTOMATIC COLLIMATOR ALIGNMENT SOFTWARE}

The semiautomatic beam-based alignment has been fully automated by closing the loop between the collimator stopping its movement after its losses exceed the threshold, and resuming the alignment based on the BLM loss signal. This involved using the feedback from the BLMs in real time to replace the user tasks (steps 1,2 and 4) with dedicated algorithms.

Cross talk analysis for parallel selection (step 1).Aligning collimators in beams 1 and 2 in parallel depends on the cross talk observed in the BLM signals. Alignments during commissioning in 2018 were done sequentially, which provided a dataset of BLM signals that were used to analyze the cross talk caused by each collimator. A dataset of 650 samples was generated, containing the BLM signals of the aligned collimators and all other collimator BLMs with losses larger than 10 times the background losses. The collimators affected by cross talk were identified by rms smoothing all BLM signals and if any BLM not attached to the moving collimator had a signal larger than $5 \%$ of the maximum loss at the aligned collimator BLM, then the collimator was labeled as having experienced cross talk. The list of collimators affected by cross talk was used as an initial model for automatically handling the parallel alignment of both beams.

Machine learning for spike detection (step 4).-The correct alignment of any collimator relies on being able to classify between alignment spikes and nonalignment spikes from the time-varying beam loss signal. This determines whether the collimator's jaws really touched the beam's reference halo, otherwise the collimator must continue moving towards the beam. Figure 6(a) shows an example of a clear alignment spike indicating that the collimator in question is aligned, whilst Fig. 6(b) shows an example of nonalignment spikes which would usually arise due to beam instabilities or mechanical vibrations. Taking a closer look at Fig. 6, a clear alignment spike consists of a steady-state signal before the spike, the loss spike itself, temporal decay of losses, and a steady-state signal after the spike. The steady

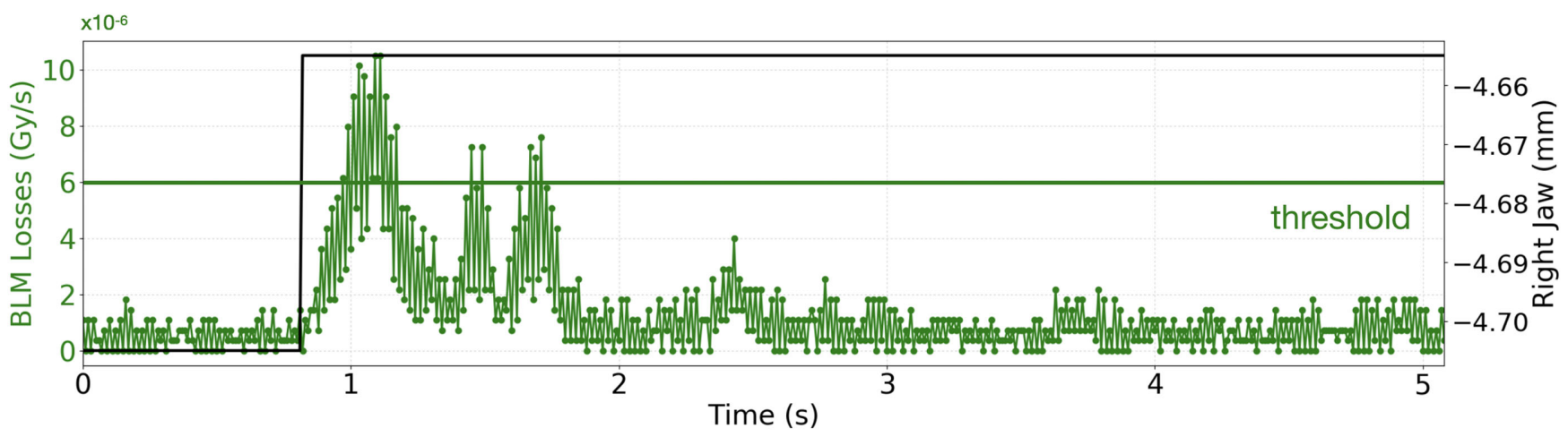

(a)

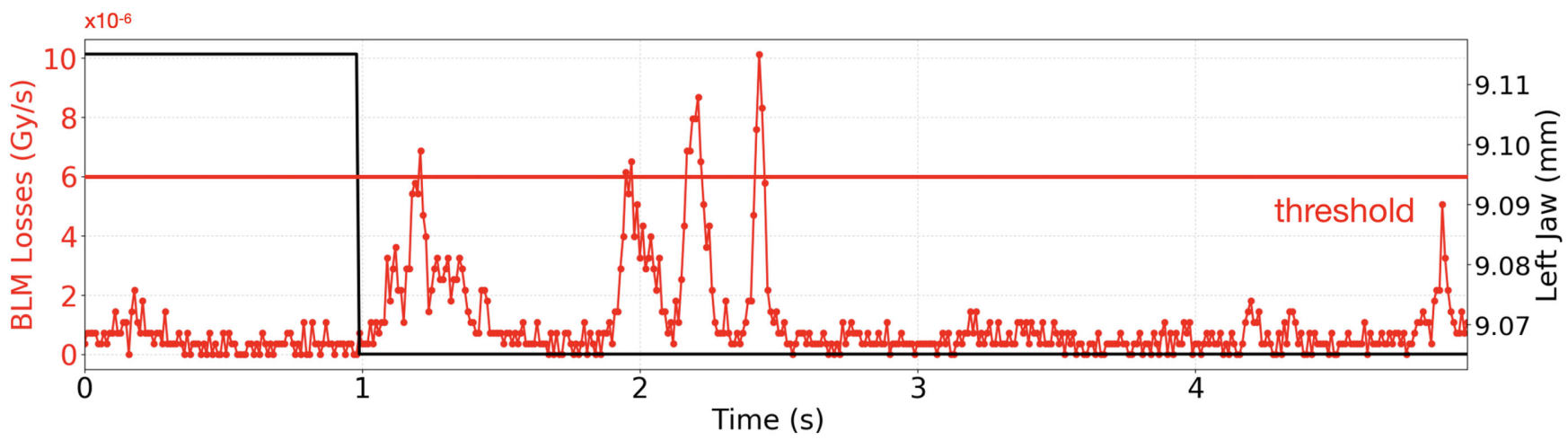

(b)

FIG. 6. Typical BLM signals as a function of time showing examples of (a) an alignment spike and (b) nonalignment spikes, after a collimator movement towards the beam [28]. 
state is a result of continuous scraping of halo particles when the jaw positions are fixed. The further a jaw cuts into the beam halo the steady-state signal increases as the density of the particles near the jaw increases. Any other spikes which do not follow this pattern are classified as nonalignment spikes; they do not have a fixed structure and can contain spurious high spikes. The fully automatic alignment software makes use of machine learning techniques to automatically classify between the two spike patterns in the BLM losses. A dataset containing 6446 samples of alignment and nonalignment spikes was assembled from previous alignment campaigns. Fourteen manually engineered features were extracted from this dataset and were analyzed. The five most important features (one feature for spike height, three features for exponential decay, one feature for collimator position) were used to train six machine learning models for comparison (logistic regression, neural network, support vector machine, decision tree, random forest, gradient boost). Each model was analyzed in depth, optimized using hyperparameters and thoroughly tested on unseen data, indicating that the support vector machine proved to be the most robust model. The suitability of using machine learning in LHC operation was confirmed during collimator alignments performed in 2018, where the machine learning models achieved a precision of over 95\% [28].
Automatic threshold selection (step 2).- -The BBA involves moving the collimators towards the beam until their losses exceed a predefined threshold, which was selected by the user based on the current BLM signal. The ideal threshold must be high enough to ignore any noisy spikes and touch the beam without interrupting the movement, and low enough to immediately stop the jaws and generate minimal losses when the collimator actually touches the beam. The algorithm for automatic threshold selection applies an exponentially weighted moving root mean square on the latest BLM signal. The thresholds selected by users for alignments in 2016 were extracted to form a dataset of 1778 samples, at injection and flattop. This dataset was used to validate the algorithm, and the difference between the thresholds selected automatically and by the user were negligible for over $90 \%$ of the cases.

\section{A. Angular collimator alignment software}

Collimators have always been aligned assuming no tilt between the collimator and the beam, as the current setting hierarchy has enough margins to safely absorb possible tilt angles. Tank misalignments or beam envelope angles at large-divergence locations could introduce a tilt, which would limit the collimation performance.

The current operational settings for the betatron cleaning hierarchy envisage a $1.5 \sigma$ retraction margin between the
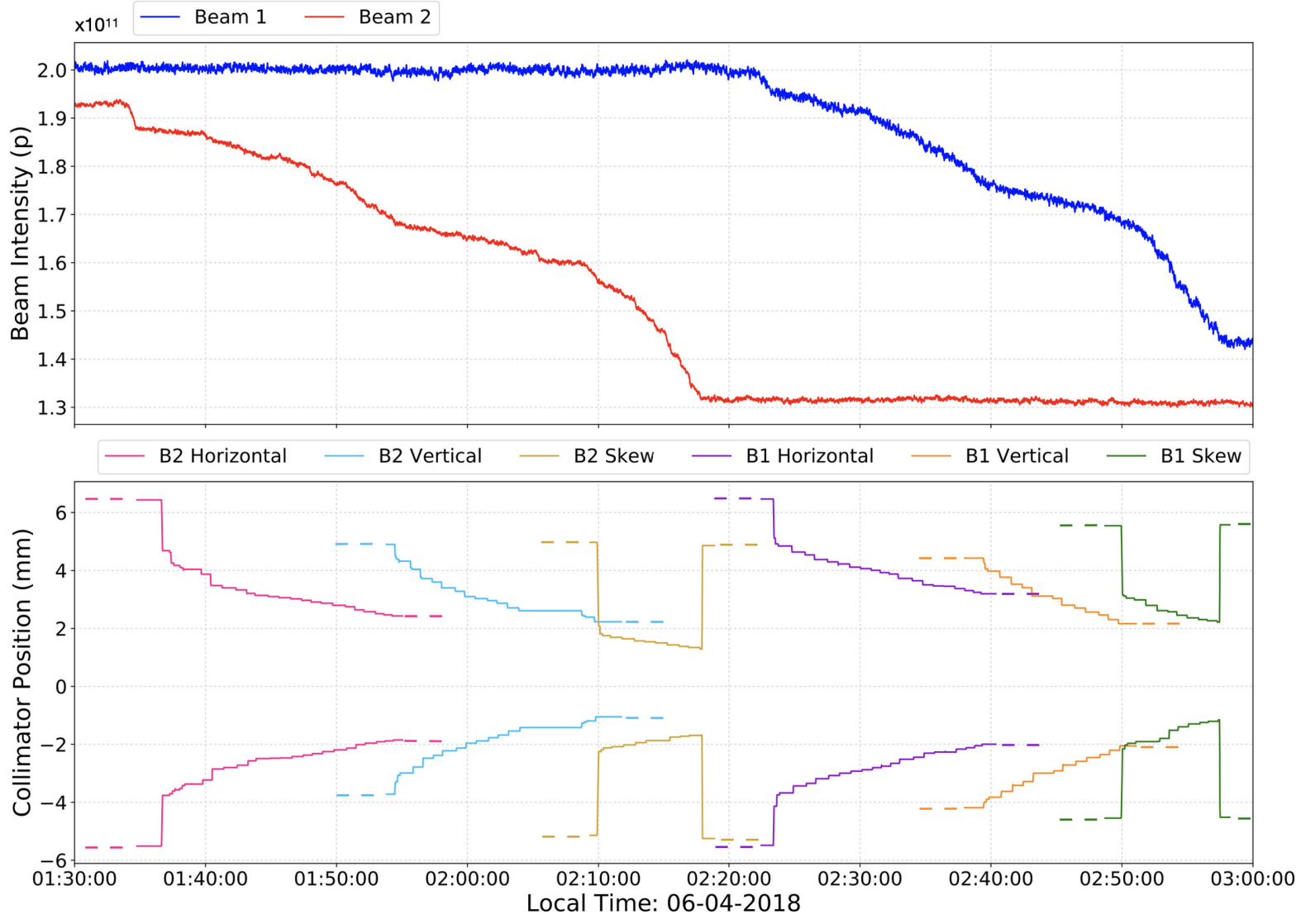

FIG. 7. Sequential alignment of the collimators in the two beams at injection, showing the beam intensities (top graph) and only the jaw positions of the primary (TCP) collimators (bottom graph) for simplicity. 
primary and the secondary collimators, which corresponds to less than $300 \mu \mathrm{m}$. In order to push the performance of the LHC, tighter collimator settings with smaller retractions are foreseen, to achieve a lower $\beta^{*}$ and improved halo cleaning [29].

A recent study [30] introduced three novel angular alignment methods to determine the most optimal angle, by applying the BBA using different tilts.

1. Angular alignment using reference collimator.-This method is based on the standard alignment procedure. A selected collimator is aligned at different angles, such that the primary collimator in the same plane is aligned before and after, to act as a reference collimator. The beam size is determined at the selected collimator at each angle, using the multiple transverse settings of the reference collimator, such that the most optimal angle is the one that resulted in the smallest beam size. This method is ideal for cases when there is an offset in the collimator tank.

2. Angular alignment at maximum angles.-This is the quickest method which aligns the selected collimator at the two maximum tilt angles $( \pm 1900 \mu \mathrm{rad})$. This provides the upstream and downstream centers which are used to calculate the optimal angle.
3. Angular alignment using a jaw as reference.-This method aligns one collimator jaw at different angles while aligning the other jaw before and after as a reference, and vice versa. This method is useful in cases of asymmetries within the collimator itself, as it is able to determine the most optimal angle for each jaw independently.

An angular alignment exploring a suitable angular range requires the equivalence of 6 to 15 standard alignments, in time. These methods made use of the semiautomatic alignment software available at the time, by applying a fixed threshold and assuming the collimators were aligned when its beam losses exceeded this threshold. As a result, a number of alignments were stopped prematurely, therefore the angular alignment software was not reliable. Therefore this angular alignment software was also integrated into the new fully automatic alignment, to prevent such misalignments in the future.

\section{RESULTS WITH PROTON BEAMS}

This section summarizes the results of the fully automatic alignment software used during 2018 LHC operation with proton beams. The first version was used during

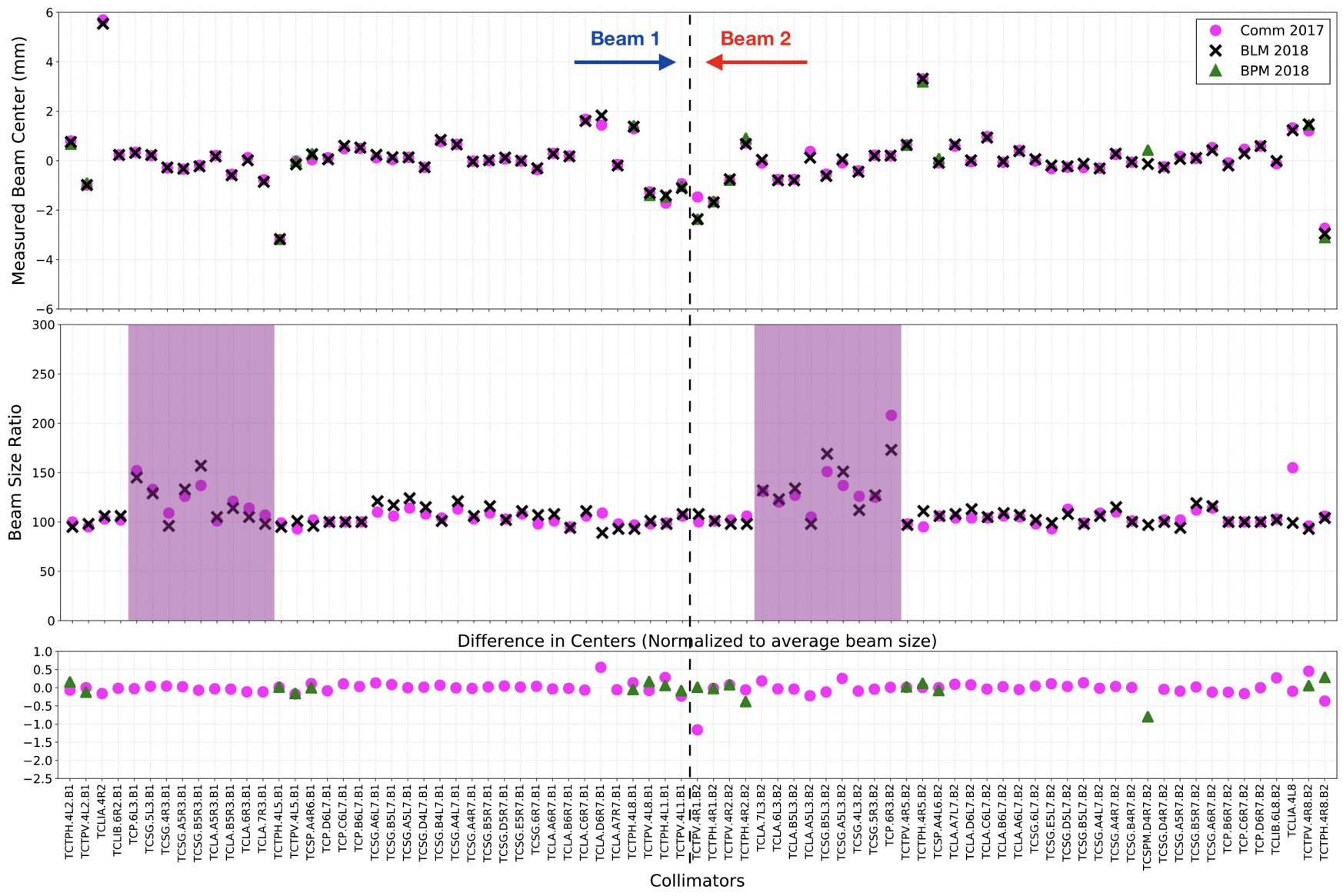

FIG. 8. Injection BLM commissioning 2018 results, comparing the beam centers to 2018 BPM centers and 2017 BLM centers (top graph), and beam size ratios compared to 2017 (middle graph). The beam size ratios in IR3 are shaded as they are expected to be large due to the higher dispersion in that location. Finally the differences between the 2018 BLM centers and the rest are compared after normalization by the average beam size of 2017 and 2018 (bottom graph). 
commissioning, whereby the software automatically aligned the collimators in the two beams sequentially. A machine development (MD) study was then scheduled to test the alignment of the two beams in parallel. Finally, another MD was scheduled to test the parallel fully automatic software with angular alignments.

\section{A. Sequential automatic alignment}

During commissioning at injection, the alignment software was used with default settings, by applying machine learning after 4 seconds of spike decay and using a 0.02 second time interval between collimator movements of $10 \mu \mathrm{m}$.

Recall from Sec. III A that the standard BBA aligns the primary collimator, of the corresponding plane, before and after each collimator is aligned. Figure 7 summarizes the alignment campaign at injection commissioning by plotting the jaw positions of the primary collimators in the horizontal, vertical and skew planes in each beam. The collimators in the two beams can be seen to be aligned sequentially with beam 2 followed by beam 1 . The entire alignment campaign required 1.5 hours for 79 collimators, therefore on average it took 1 minute to align a single collimator using this approach.

The collimator centers measured at injection with BLM detectors are similar to the centers obtained during injection commissioning in 2017, and are consistent with the centers measured using BPMs (where available), as shown in Fig. 8. The beam sizes are also consistent with 2017, evidently showing the reproducibility of the LHC after correction of orbit and optics. Furthermore, the difference in centers is normalized to the average beam size of 2017 and 2018, to better compare the centers across the LHC ring.

During commissioning at flattop, the alignment conditions were difficult due to beam stability issues, especially in the horizontal plane in beam 1 . Due to this, the settings of the fully automatic tool were modified to be more conservative with collimator movements to limit high losses. This was performed by applying machine learning after 6 seconds of spike decay and using a 0.2 second time interval between collimator movements of $5 \mu \mathrm{m}$. Using these settings, the 75 collimators were aligned in 3.5 hours therefore it took an average of 2 minutes to align a single collimator using this approach. The entire alignment campaign is displayed in Fig. 9, showing that the horizontal plane in beam 1 took the most time to align. Similar to injection, the flattop beam centers and sizes measured with BLMs and BPMs (where available) are consistent between 2017 and 2018, as shown in Fig. 10.

These commissioning campaigns at injection and flattop were validated by generating loss maps (refer to the

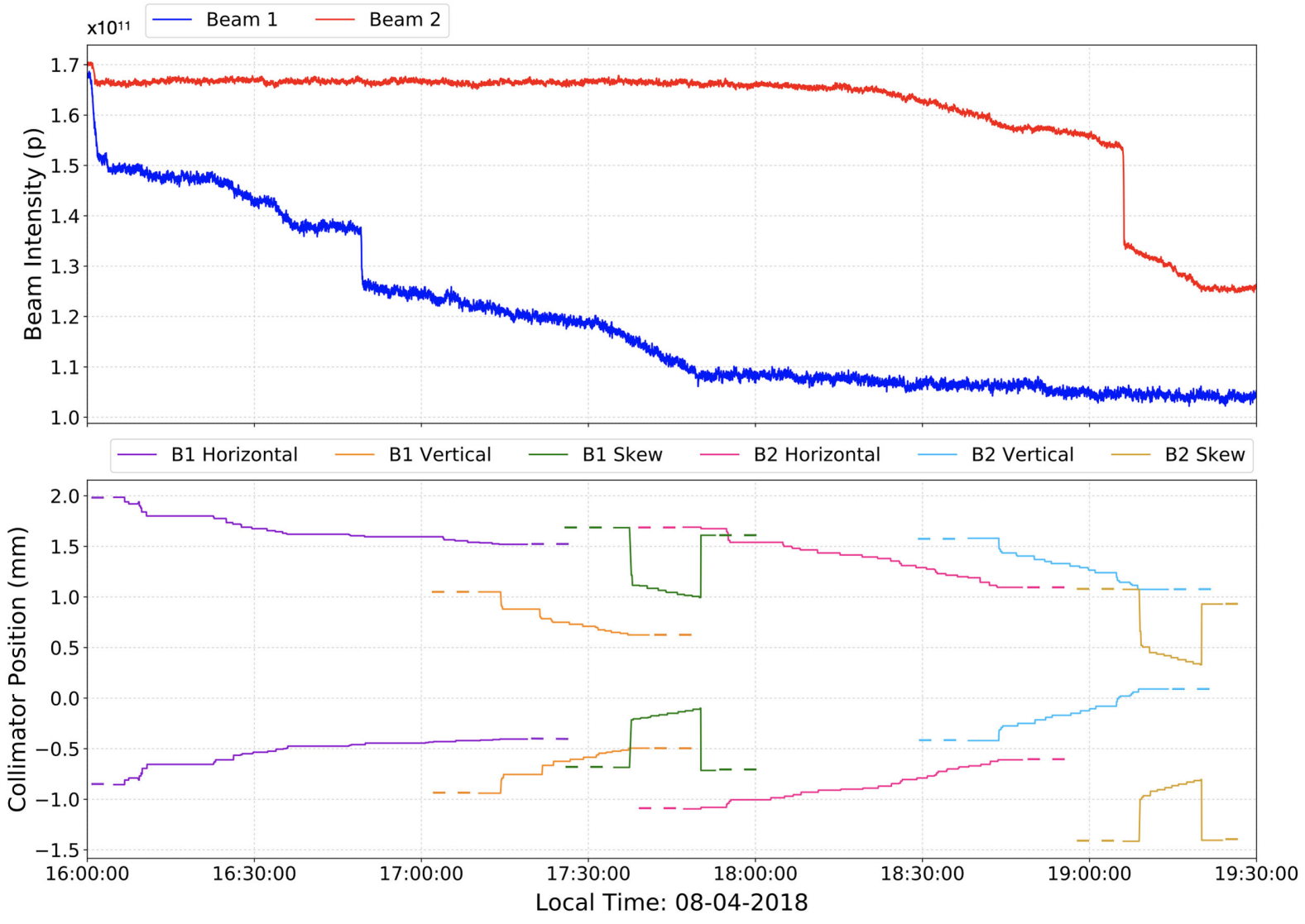

FIG. 9. Sequential alignment of the collimators in the two beams at flattop, showing the beam intensities and only the jaw positions of the primary (TCP) collimators for simplicity. 


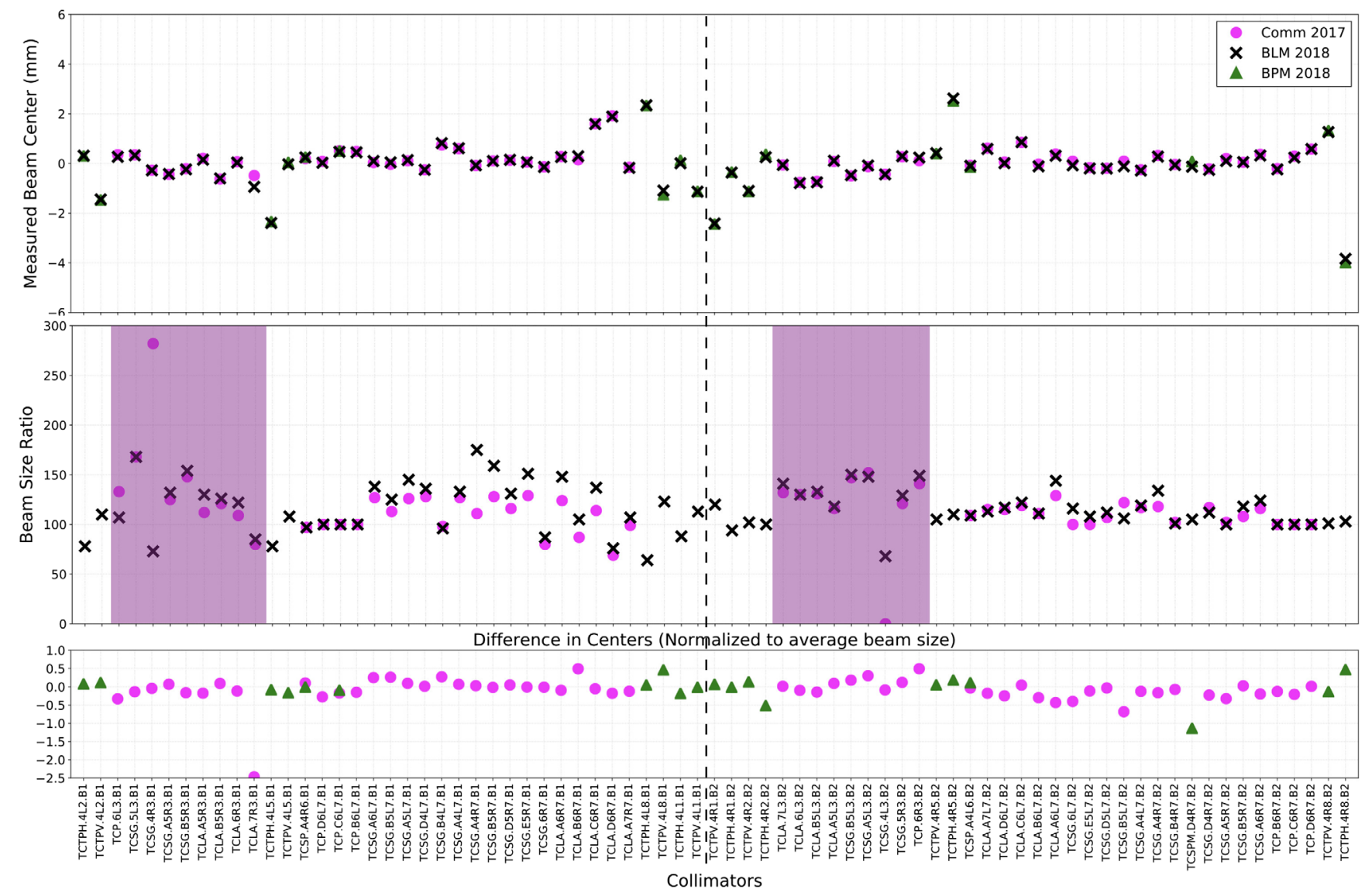

FIG. 10. Flattop BLM commissioning 2018 results, comparing the beam centers to 2018 BPM centers and 2017 BLM centers (top graph), beam size ratios compared to 2017 (middle graph), and comparison of the difference in centers normalized by the average beam size (bottom graph).

Appendix), which are summarized by observing the losses in the dispersion suppressor in IR7. These losses determine the collimation inefficiency, and Fig. 11 compares the inefficiency between 2017 and 2018. The difference is within an acceptable limit, as any misalignments would result in a factor of 2 to 3 difference [31]. Therefore the results are consistent, thus validating both alignment campaigns.

\section{B. Parallel automatic alignment}

During an MD at injection, a second version of the fully automatic alignment software was tested [32]. The default injection settings were used for the alignment software, i.e., applying machine learning after 4 seconds of spike decay and using a 0.02 second time interval between collimator movements of $10 \mu \mathrm{m}$. The two beams were aligned in

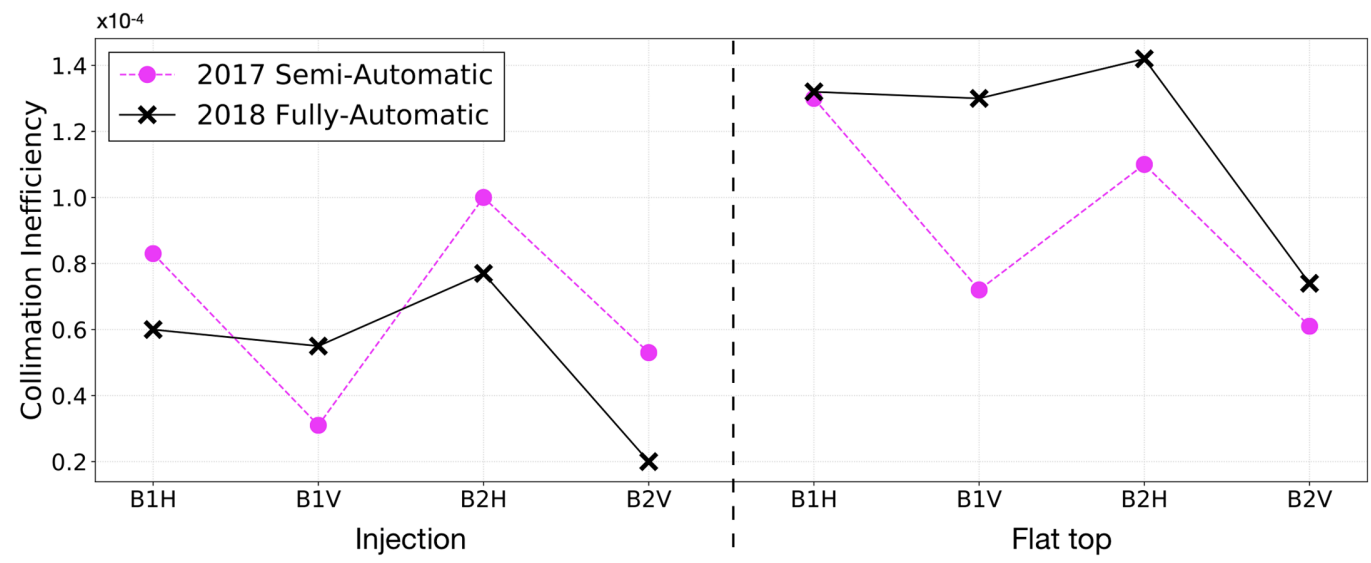

FIG. 11. Comparison of losses in the IR7 DS after commissioning using the fully automatic alignment in 2018 and the semiautomatic in 2017, extracted from [31]. 

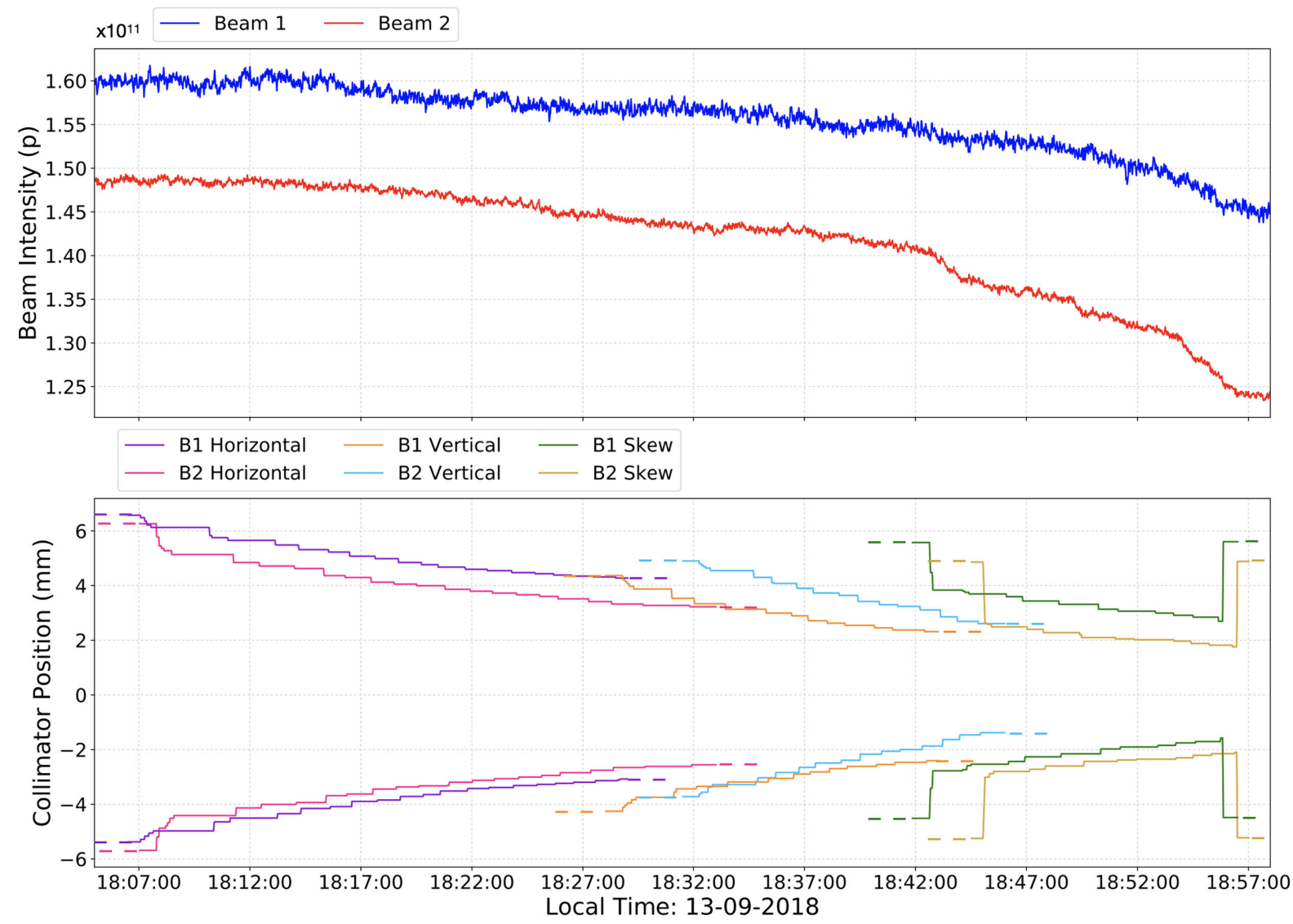

FIG. 12. Parallel alignment of the collimators in the two beams at injection, showing the beam intensities and only the jaw positions of the primary (TCP) collimators for simplicity.

parallel and the entire alignment campaign is displayed in Fig. 12. The alignment of 79 collimators at injection took 50 minutes, decreasing the alignment time at injection by a factor of 3, with respect to 2017 as shown in Fig. 13. On average it took 1.5 minutes to align two collimators using this approach.

The beam centers and beam sizes measured at each collimator are consistent with those from commissioning 2018, as shown in Fig. 14. This validates the new parallel alignment software, confirming that it can be used as a baseline for future parallel alignments at injection, and should be repeated for flattop data.

In the future it is planned for this software to be further upgraded based on more in-depth cross talk analysis studies [33].

\section{Angular automatic alignment}

The three angular alignment methods introduced in a previous MD [34] were used to align a number of collimators using the semiautomatic software. In 2018, the parallel fully automatic alignment was incorporated into the angular alignment software, and the angular alignments of the same collimators were repeated in an MD at injection [35]. Each collimator was first aligned with BPMs, then an angular range between $-600 \mu \mathrm{rad}$ and $0 \mu \mathrm{rad}$ was explored, using an angle step size of $50 \mu \mathrm{rad}$.

As an example, Fig. 15 shows the results obtained for the TCTPH.4R2.B2, a tertiary collimator in the horizontal plane on the right of IR2 in beam 2. Each method converges to an angle of approximately $-370 \mu \mathrm{rad}$, indicating there is a real offset of the collimator tank, which is consistent with the angle found in the previous MD.

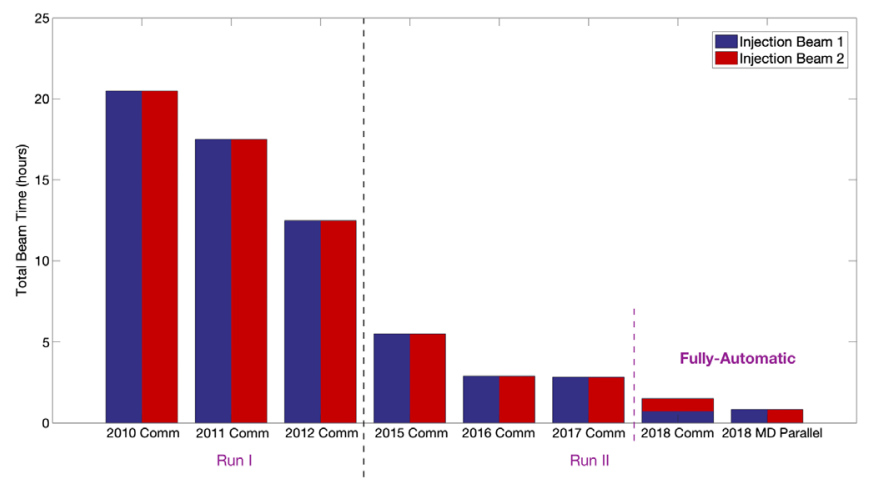

FIG. 13. The time to align 79 collimators at injection commissioning, compared to the 2018 parallel alignment MD. 


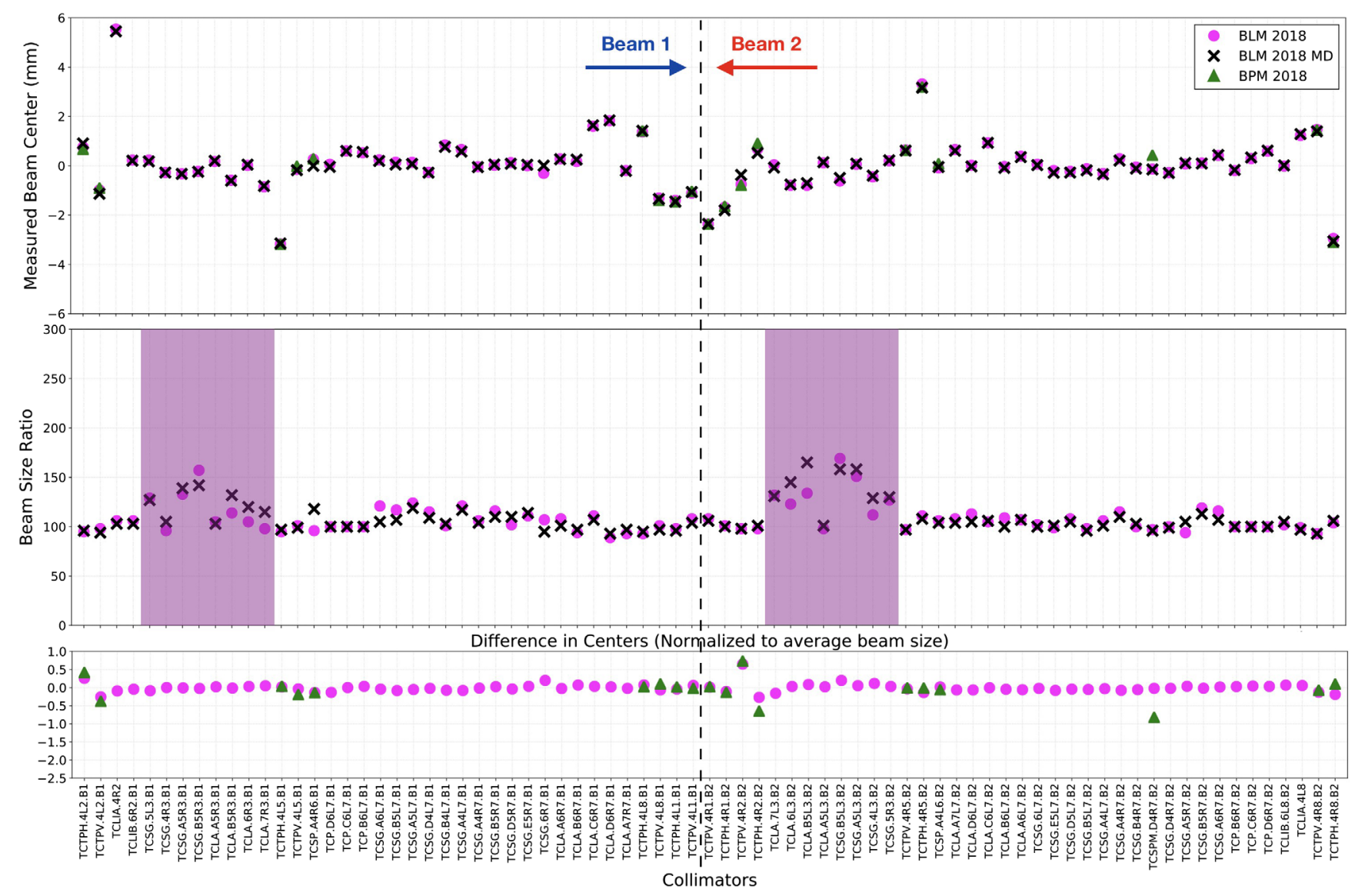

FIG. 14. Parallel alignment MD results at injection, comparing the beam centers (top graph) and beam size ratios (middle graph) to the 2018 injection commissioning results using BLMs and BPMs. The difference in centers normalized by the average beam size is presented on the bottom graph.

Figure 16 displays the results for the TCSG.D4L7.B1, a secondary collimator in the vertical plane on the left of IR7 in beam 1 . These results indicate an angle of $-500 \mu \mathrm{rad}$ for the left jaw and $300 \mu \mathrm{rad}$ for the right jaw. This suggests an asymmetry within the collimator itself, which is also possible and consistent with previous results.

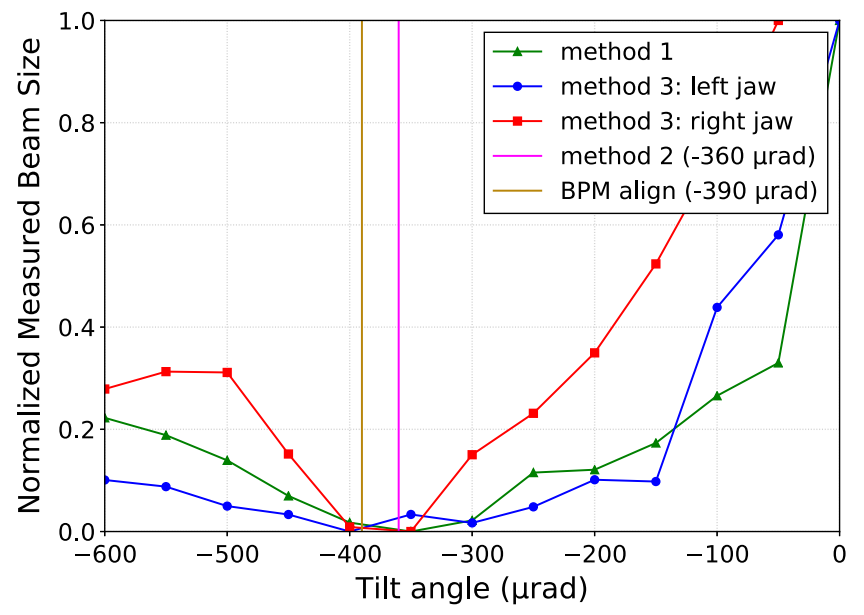

FIG. 15. Angular alignment results for the TCTPH.4R2.B2 using the three methods and the BPMs.
On average it took 12 minutes to align a single collimator at 12 different angles using method 1,2 minutes to align at three different angles using method 2, and 13 minutes to align at 24 different angles using method 3. Overall, the fully automatic tool aligned collimators with minimal misalignments, and the angles obtained are consistent with

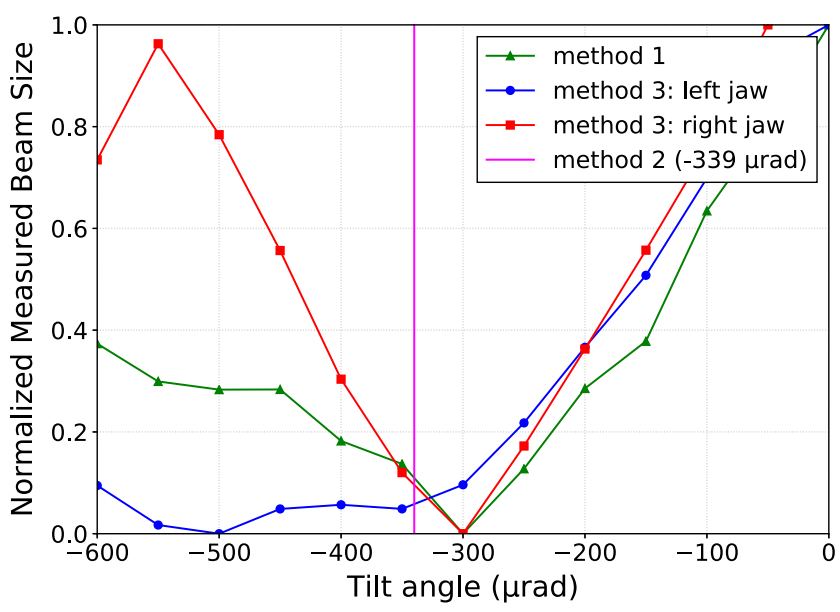

FIG. 16. Angular alignment results for the TCSG.D4L7.B1 using the three methods. 
previous alignments. These results indicate that the new angular alignment software is reliable and that the results are reproducible. This software may be used in the near future, if a tighter hierarchy needs to be achieved.

\section{RESULTS WITH ION BEAMS}

During ion beam commissioning in 2018, the fully automatic alignment software was used to align all IR7 collimators in collisions. The losses generated by ion beams exceed the proton losses by a factor 100 in the dispersion suppressor (DS) [36]. As a consequence, the fully automatic tool was modified to avoid high DS losses by using a 0.1 second time interval between collimator movements of $10 \mu \mathrm{m}$ and aligning the collimators in the two beams sequentially. On average it took 1.5 minutes to align a single collimator using this approach.

Since no heavy ion run took place in 2017, there is no data available to compare to the 2018 alignment. However the collimators in IR7 are not expected to have large changes between collisions with ion beams and flattop with proton beams (recall Sec. V). Therefore this comparison of centers is displayed in Fig. 17, and the results are consistent for the majority of the collimators. A couple of collimators

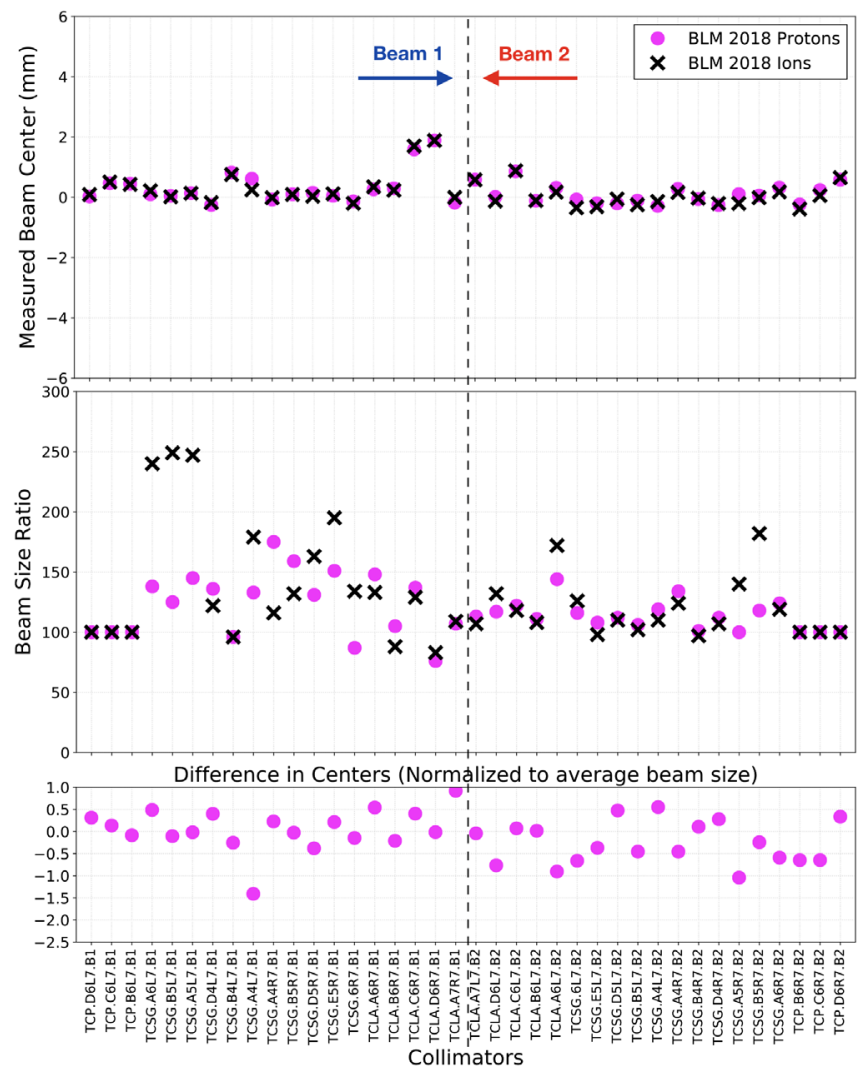

FIG. 17. Ion commissioning IR7 BLM results in collisions comparing the beam centers and beam size ratios to flattop commissioning results. indicate a difference between \pm 150 and $\pm 200 \mu \mathrm{m}$, which could be expected due to the change of reference orbit between the two beam types.

\section{CONCLUSION}

The LHC is protected by a complex collimation system, having collimators aligned with the beam to form a hierarchy. Alignments are performed at the start of each year during commissioning, and may be repeated when the machine parameters are changed, therefore it is important to be efficient while maintaining reliable results. During the past years, BLM-based collimator alignments have been performed semiautomatically, requiring collimation experts to oversee and control the alignment.

Recent work sought to apply machine learning and other algorithms based on BLM signal analysis to transform the alignment from semiautomated to fully automated. This new fully automatic software was used in all collimator alignments throughout 2018, and this paper presents the results obtained for various alignment campaigns. The first version was used during commissioning, such that the collimators in the two beams were automatically aligned sequentially, at injection and flattop. A few months later an improved version of the software was used to successfully align the collimators in the two beams in parallel at injection by making use of a new cross talk model. This successfully decreased the alignment time by a factor of 3 at injection, as compared to the 2017 data.

Finally, this fully automatic tool was also incorporated into the angular alignment software and produced reliable results, when tested on a few controlled cases. Overall, the full automation with the use of machine learning has proven to be more efficient and able to generate reproducible results. Therefore, the plan is to use this as the default alignment software for starting LHC operation in 2021.

\section{ACKNOWLEDGMENTS}

The authors would like to thank the collimation team and the controls team for their assistance during commissioning and MDs, as well as the operations teams for assisting during the beam studies.

\section{APPENDIX: COMMISSIONING 2018 LOSS MAPS}

The alignment campaigns during commissioning at the start of each year are followed by loss maps to validate the collimation hierarchy defined by the alignment. The loss maps generated after injection and flattop commissioning in 2018 are displayed in Figs. 18 and 19, respectively. Loss maps are individually generated for the horizontal and vertical planes in each beam. One can see that in each of the four cases the collimators absorb the majority of the losses and these losses follow the direction of the beam, indicating the correct hierarchy. 

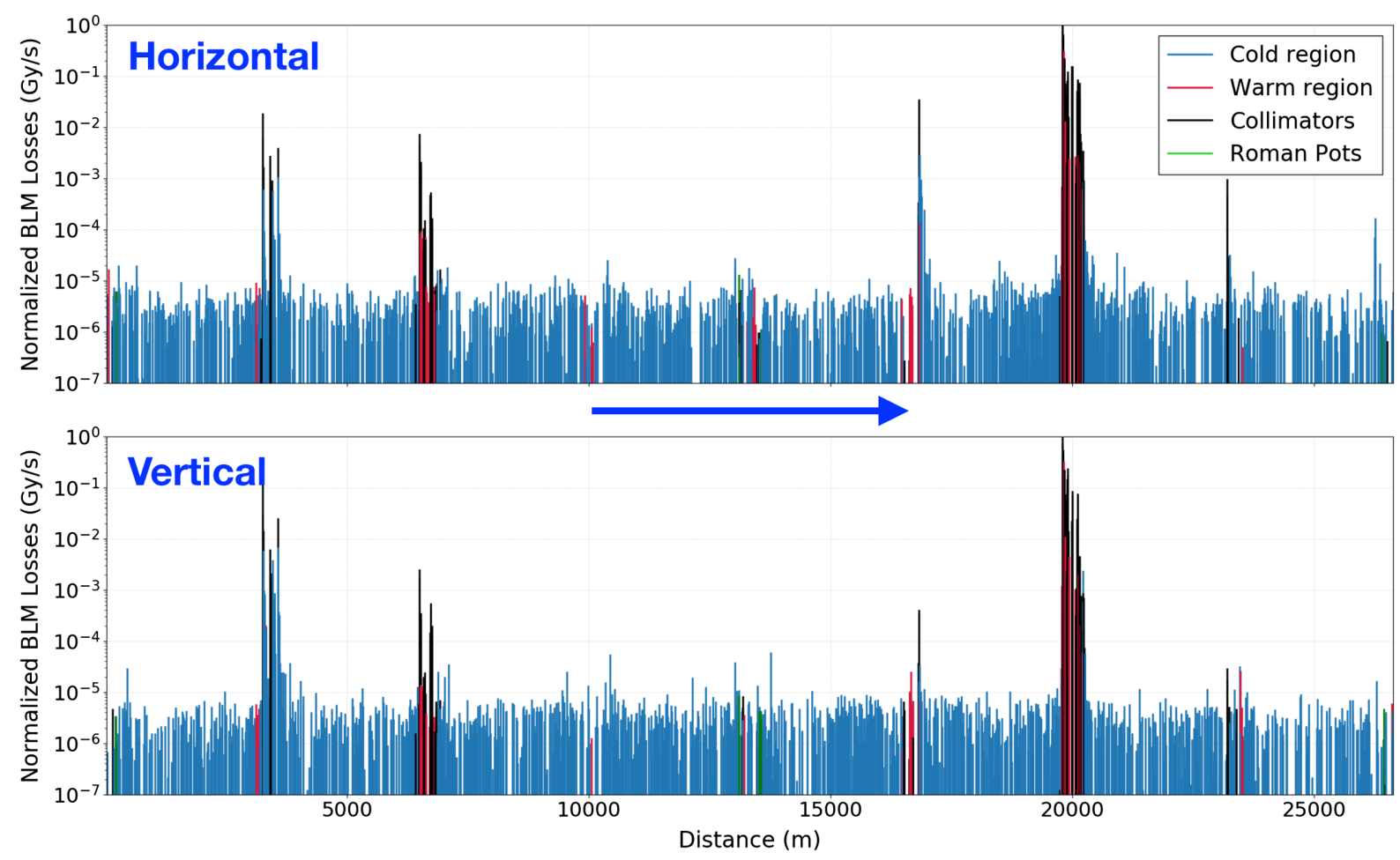

(a)
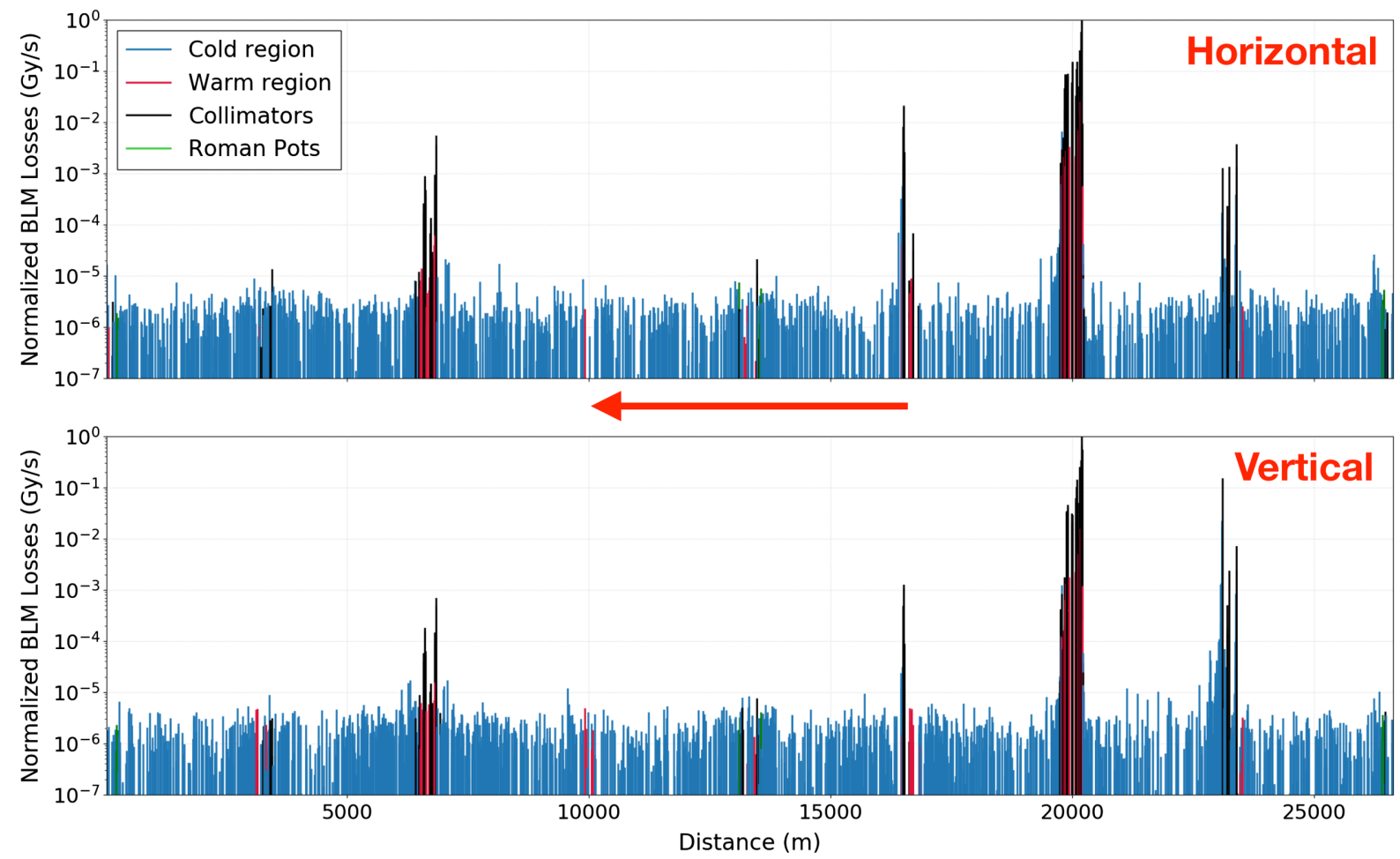

(b)

FIG. 18. Loss maps generated on 13-04-2018 to validate the BLM collimator alignment at injection for (a) beam 1, and (b) beam 2, indicating the beam direction by the arrow in each case. 

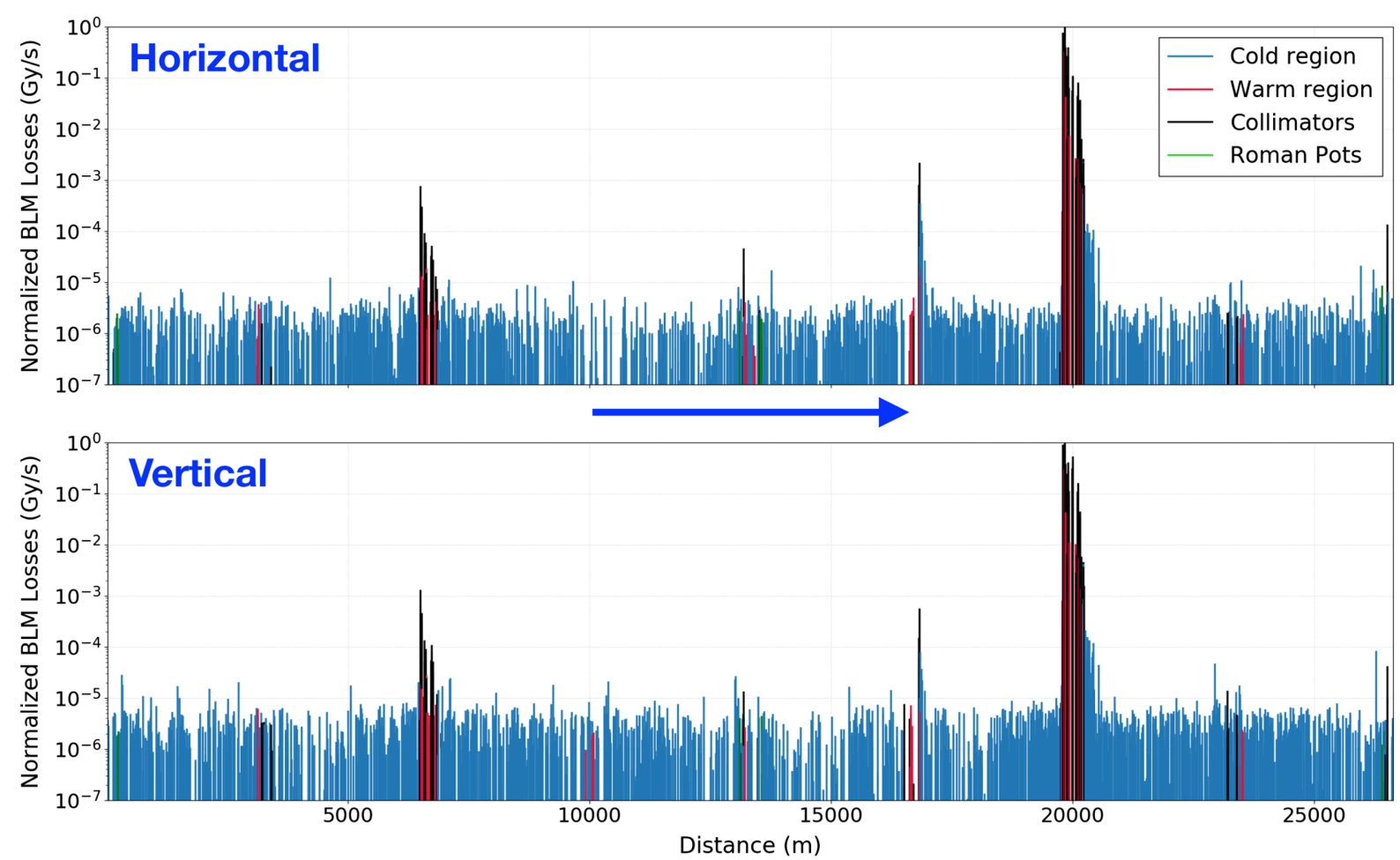

(a)
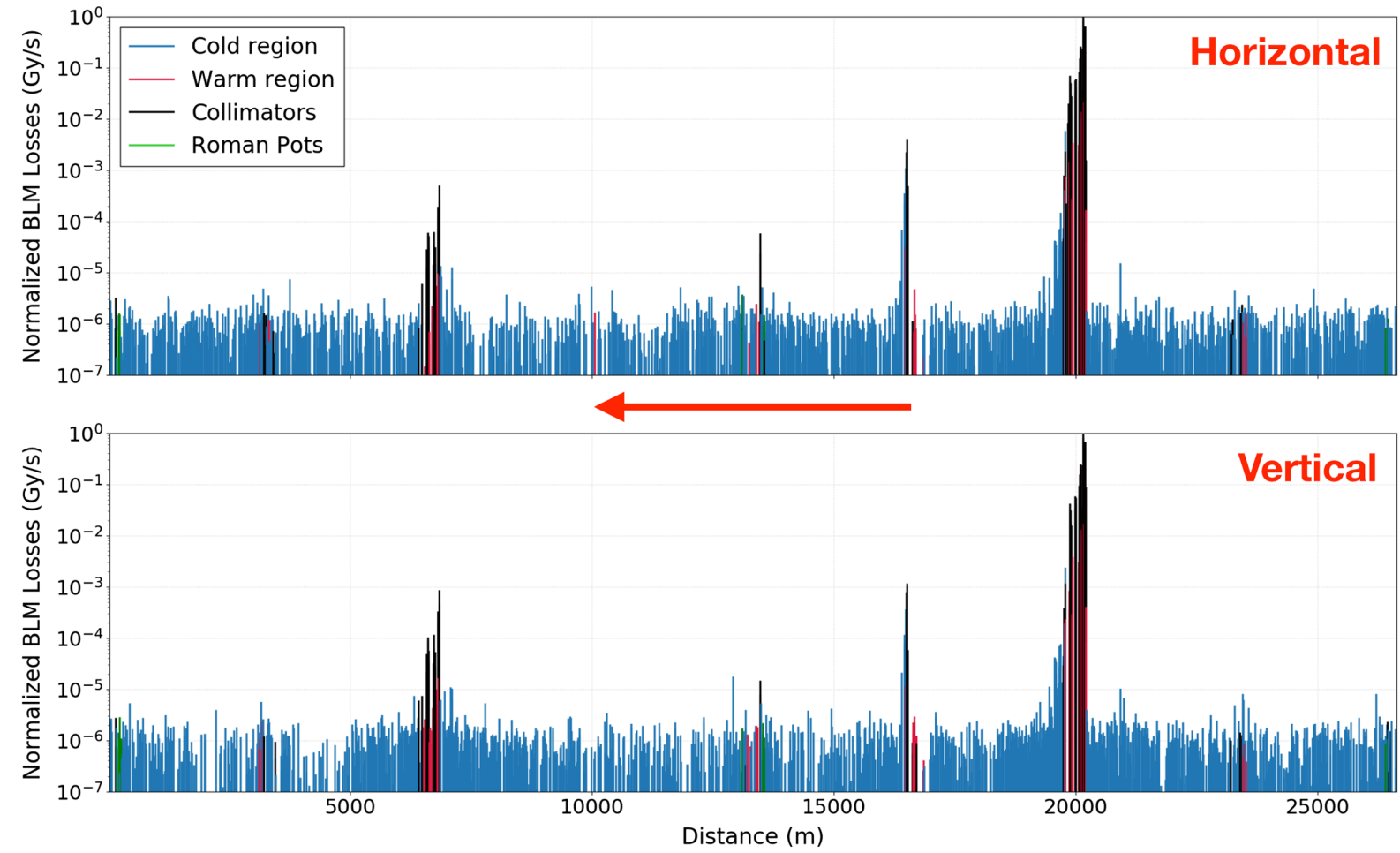

(b)

FIG. 19. Loss maps generated on 13-04-2018 to validate the BLM collimator alignment at flattop for (a) beam 1, and (b) beam 2, indicating the beam direction by the arrow in each case. 
[1] L. Evans, The Large Hadron Collider, New J. Phys. 9, 335 (2007).

[2] L. Evans and P. Bryant, LHC machine, J. Instrum. 3, S08001 (2008).

[3] G Aad et al., The ATLAS experiment at the CERN large hadron collider, J. Instrum. 3, S08003 (2008).

[4] K Aamodt et al., The ALICE experiment at the CERN LHC, J. Instrum. 3, S08002 (2008).

[5] S. Chatrchyan et al., The CMS experiment at the CERN LHC, J. Instrum. 3, S08004 (2008).

[6] A. Alves, Jr. et al., The LHCb detector at the LHC, J. Instrum. 3, S08005 (2008).

[7] L. R. Evans, Beam physics at LHC, in Proceedings of the 2003 Particle Accelerator Conference, Portland, OR (IEEE, New York, 2003), Vol. 1, pp. 19-23.

[8] R. W. Aßmann et al., Requirements for the LHC collimation system. Technical report, 2002.

[9] S. Redaelli, R. W. Assmann, R. Bruce, A. Rossi, and D. Wollmann, Operational performance of the LHC collimation, in HB2010, Morschach, Switzerland (2010), pp. 395-399, http://accelconf.web.cern.ch/AccelConf/ HB2010/papers/tuo2c05.pdf.

[10] D. Missiaen, R. J. Steinhagen, and J.-P. Quesnel, The alignment of the LHC, Technical report, 2009.

[11] O. Brüning, LHC Design Report: The LHC Main Ring, European Organization for Nuclear Research (CERN, Geneva, 2004), Vol. 1.

[12] D. Wollmann et al., First cleaning with LHC collimators, Technical report, 2010.

[13] E. Holzer et al., Beam loss monitoring system for the LHC, in Nuclear Science Symposium Conference Record, 2005 (IEEE, New York, 2005), Vol. 2, pp. 1052-1056.

[14] M. Stockner et al., Classification of the LHC BLM ionization chamber, Technical report, 2007.

[15] G. Valentino et al., Final implementation, commissioning, and performance of embedded collimator beam position monitors in the Large Hadron Collider, Phys. Rev. Accel. Beams 20, 081002 (2017).

[16] M. Lamont, The LHC from commissioning to operation, Proceedings of IPAC2011, San Sebastian, Spain, MOYAA01 (2011), http://accelconf.web.cern.ch/AccelConf/ IPAC2011/papers/moyaa01.pdf.

[17] A. Mereghetti et al., Performance of the collimation system during 2016-hardware perspective, in the 7th Evian Workshop on LHC Beam Operation, Evian, France (CERN, Geneva, 2017), pp. 225-228.

[18] R. Bruce, S. Redaelli, and R. W. Aßmann, Principles for generation of time-dependent collimator settings during the LHC cycle, in Proceedings of the 2nd International Particle Accelerator Conference, San Sebastián, Spain, THPZ029 (2011), http://accelconf.web.cern.ch/AccelConf/ IPAC2011/papers/THPZ029.PDF.

[19] R. J. Steinhagen, LHC beam stability and feedback controlorbit and energy, Ph.D. thesis, RWTH Aachen University, 2007.

[20] B. Salvachua et al., Observations of beam losses at the LHC during reduction of crossing angle, in Proceedings of
IPAC17, Copenhagen, Denmark, No. IPAC-2017TUPVA025, 2017.

[21] G. Valentino, A. A. Nosych, R. Bruce, M. Gasior, D. Mirarchi, S. Redaelli, B. Salvachua, and D. Wollmann, Successive approximation algorithm for beam-positionmonitor-based LHC collimator alignment, Phys. Rev. ST Accel. Beams 17, 021005 (2014).

[22] R.W. Aßmann et al., Expected performance and beambased optimization of the LHC collimation system, Technical report, 2004.

[23] G. Robert-Demolaize, Design and performance optimization of the LHC collimation system, Technical report, 2006.

[24] S. Redaelli et al., Operational experience with a LHC collimator prototype in the CERN SPS, Technical report, 2009.

[25] G. Valentino, S. Redaelli, R. W. Aßmann, N. Sammut, and D. Wollmann, Semiautomatic beam-based alignment algorithm for the LHC collimation system, Technical report, 2011.

[26] G. Valentino, R. Aßmann, R. Bruce, S. Redaelli, A. Rossi, N. Sammut, and D. Wollmann, Semiautomatic beam-based LHC collimator alignment, Phys. Rev. ST Accel. Beams 15, 051002 (2012).

[27] G. Valentino et al., Fast automatic beam-based alignment of the LHC collimation system, in the 14th International Conference on Accelerator \& Large Experimental Physics Control Systems (ICALEPCS2013), San Francisco, California (2013), pp. 6-11, http://accelconf.web.cern .ch/AccelConf/ICALEPCS2013/papers/thcocb03.pdf.

[28] G. Azzopardi, G. Valentino, A. Muscat, and B. Salvachua, Automatic spike detection in beam loss signals for LHC collimator alignment, Nucl. Instrum. Methods Phys. Res., Sect. A 934, 10 (2019).

[29] A. Mereghetti et al., $\beta^{*}$-reach-IR7 collimation hierarchy limit and impedance, Technical report, 2016.

[30] G. Azzopardi et al., Automatic angular alignment of LHC collimators, in the 16th International Conference on Accelerator \& Large Experimental Physics Control Systems (ICALEPCS2017), Barcelona, Spain (2017), pp. 928-933.

[31] N. Fuster-Martínez et al., Run 2 collimation overview, in the 9th Evian workshop on LHC beam operation, Evian, France (to be published).

[32] G. Azzopardi, B. Salvachua Ferrando, and G. Valentino, MD3343-fully automatic parallel collimation alignment using machine learning, Technical report, 2018.

[33] G. Azzopardi, B. Salvachua Ferrando, and G. Valentino, Data-driven cross-talk modeling of beam losses in LHC collimators, Phys. Rev. Accel. Beams 22, 083002 (2019).

[34] G. Azzopardi et al., Automatic angular alignment of LHC collimators, Technical report, 2017.

[35] G. Azzopardi, B. Salvachua Ferrando, and G. Valentino, MD3344-fully automatic angular alignment of LHC collimators using machine learning, Technical report, 2018.

[36] D. Mirarchi et al., Collimation: Experience and performance, in the 7th Evian Workshop on LHC Beam Operation, Evian, France (CERN, Geneva, 2017). 